\title{
ERGONOMICS OF SELECTED LAPAROSCOPIC PROCEDURES - NEED FOR ACTION?
}

\author{
Pfeffer $\mathrm{S}^{1}$, Hofmann $\mathrm{A}^{1}$, Maier $\mathrm{T}^{1}$, Rothmund $\mathrm{R}^{2}$, Sievert $\mathrm{K}-\mathrm{D}^{3}$, Seibt $\mathrm{R}^{4}$, Rieger MA ${ }^{4}$, Steinhilber $\mathrm{B}^{4}$ \\ ${ }^{1}$ Institute of Engineering Design and Industrial Design, University of Stuttgart, Germany \\ ${ }^{2}$ University Department of Gynecology and Obstetrics, University Hospital Tuebingen (UKT), Germany \\ ${ }^{3}$ University Department of Urology, UKT, Germany \\ ${ }^{4}$ Institute of Occupational and Social Medicine and Health Services Research, UKT, Germany \\ stefan.pfeffer@iktd.uni-stuttgart.de
}

\begin{abstract}
Objective of the study was a comprehensive description of selected standard laparoscopic interventions in urology and gynecology by a multiple measurement approach using subjective and objective assessment techniques. The results show substantial asymmetric biomechanical strain (sEMG) which could not be observed by subjective assessment techniques and feedback of the surgeons.
\end{abstract}

Keywords: Laparoscopy, Workload, NASA TLX, sEMG

\section{Introduction}

Since laparoscopic surgery became a common intervention at the beginning of the 1990s human factors research concerning this workplace started almost simultaneously and revealed situations of poor ergonomics at that time [1], [2]. More than 20 years later we ask whether things have improved. Despite innovations in surgical equipment and highly developed procedures in robotic surgery, recent surveys among surgeons report about physical discomfort due to suboptimal working postures [3]. In order to develop strategies to improve ergonomic conditions of laparoscopic surgery a detailed description of such workplaces is required using multiple measurement approach [4]. We therefore observed two operating departments at the UKT to evaluate the status quo.

\section{Methods}

The multiple measurement approach provided subjective questionnaire data (NASA TLX [5], Nordic Questionnaire [6]), objective measurement data (sEMG, ECG and posture sensors), and life record data (3-perspective videoanalysis). In total 16 standard surgical interventions with comparable laparoscopic procedures were observed in the urological clinic (prostatectomy, nephrectomy and cystectomy; $n=6$ ) and the gynecological clinic (hysterectomy, ovariectomy; $\mathrm{n}=10$ ). These interventions were accomplished by six experienced surgeons (work experience $>10$ years) and took place between 8 a.m. and 3 p.m. with a duration of 20 to 180 minutes (1-4 interventions a day).

Figure 1 shows the setting of the urological operating room (OR). Surgeons' movements and postures were captured by three cameras recording the legs and feet (camera 1), the torso, arms and head in front (camera 2) and lateral view (camera 3).

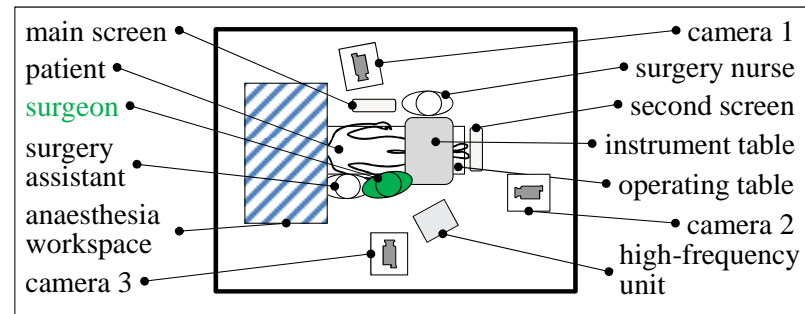

Figure 1: Setting urological OR

Accelerometer based posture sensors were placed at the back and on the upper arm. These sensors continuously measured the arm abduction/anteversion and the torso forward/backward flexion and lateral flexion. Biomechanical strain was quantified by surface electromyography (sEMG) to perform activity analysis of the lower back (M. erector spinae left \& right), the neck (M. trapezius pars descendens left \& right) and the shoulder of the dominant arm (M. deltoideus pars clavicularis, pars acromialis \& spinalis). SEMG activity and ECG (heart rate) were determined continuously throughout the laparoscopic interventions. SEMG was normalized to a submaximal static reference contraction with an external load of $2 \mathrm{~kg}$. ECG was recorded by single channel measurement.

After each intervention the surgeons had to complete the NASA TLX workload weighting and rating. The Nordic Questionnaire gave information about surgeons' musculoskeletal health status in general.

\section{Results}

Subjective questionnaire data: NASA TLX evaluation showed an Overall Weighted Workload Score (OWWS) of middle load for both types of intervention (see Fig. 2).

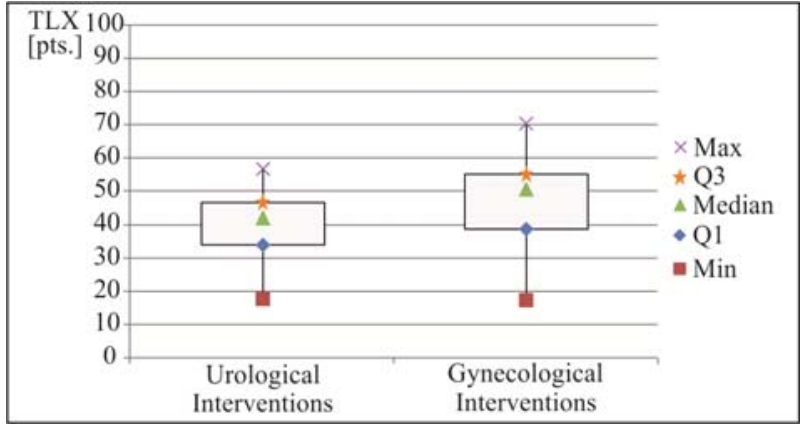

Figure 2: Overall Weighted Workload Score (NASA TLX) 
Attention should be paid to the single dimension of physical demand which represented only 9.2\% (gynecological interventions) resp. 9.9\% (urological interventions) of OWWS.

An ABC priority analysis of the answers given in the Nordic questionnaire showed that surgeons mostly had health problems in the shoulder region (A), the neck and lower back region (B) and the hip (C). These results are comparable to the survey of Miller et al. [3].

Objective test data: Figure 3 and 4 show the normalized sEMG activity of the trapezius muscles and the arm abduction in degrees $\left[^{\circ}\right.$ ] of a single subject representative for the studied sample. SEMG indicated asymmetric biomechanical strain with significantly higher muscular activity in the neck of the dominant arm, operating the laparoscope (see Figure 3). Posture sensors indicate high amounts of arm abduction (see Figure 4). No remarkable events were observed in the course of the ECG signal.

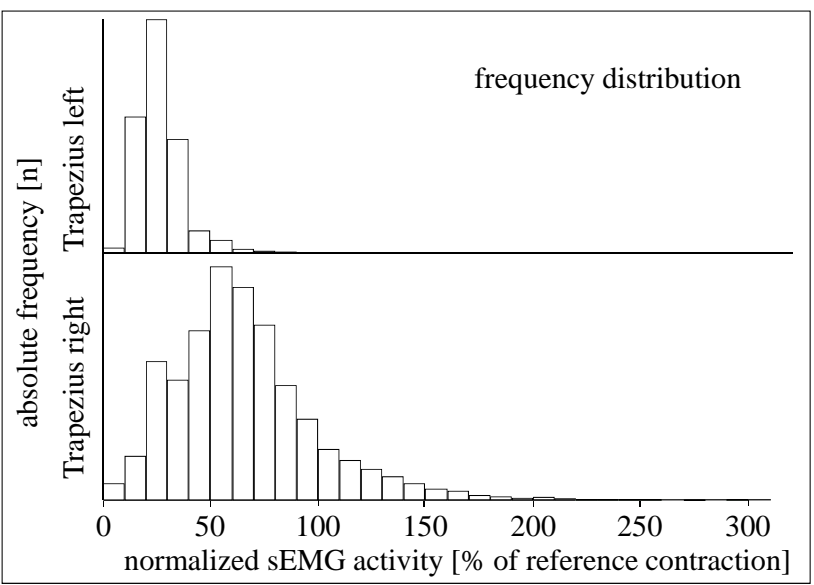

Figure 3: Electric activity of M. trapezius (normalized sEMG)

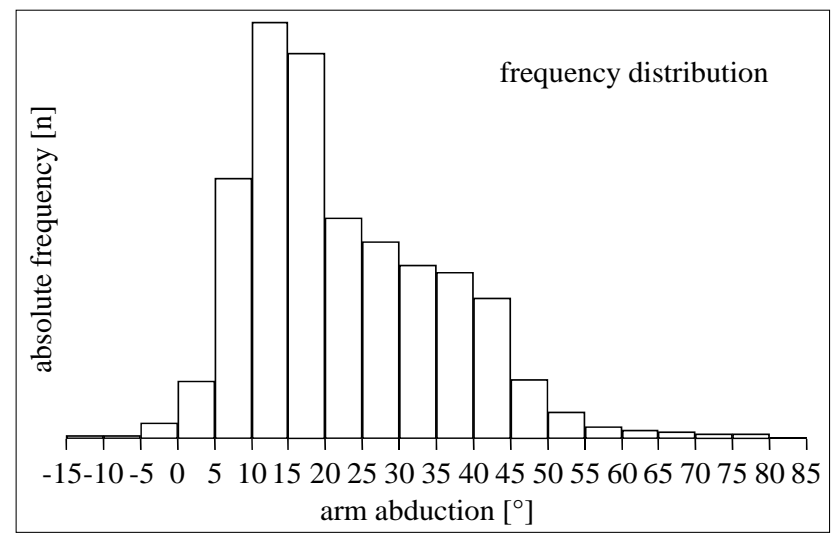

Figure 4: Arm abduction (posture sensor)

Life record data: Videoanalysis provided setting-related, task-related, and individual factors influencing surgeons' postures. Settings with the main screen positioned above eye level caused surgeons to tilt their head backwards. Task-related changes in posture force surgeons to flex and rotate their body to keep the screen in sight. Surgeons thereby showed individual postures despite similar settings and tasks as well as individual ways to support their posture, such as leaning against the operating table or resting their arms on the table.

\section{Discussion}

Although surgeons' subjective ratings of physical demands during laparoscopic surgery were low, as indicated by the NASA TLX questionnaire, asymmetric biomechanical strain was identified by SEMG and video analysis. It seems reasonable that biomechanical strain of a single laparoscopic surgery is uncritical, however cumulative biomechanical strain summarized by months and years of laparoscopic surgery may have a substantial effect on the risk of developing musculoskeletal diseases. This appraisal corresponds to the results of the Nordic questionnaire which showed a prevalence of health problems in the shoulder region of up to more than 30 days during the last 12 months. We therefore conclude that laparoscopic surgery should be considered as a physical demanding task. Thus, ergonomic improvements are necessary to reduce the risk of developing musculoskeletal diseases after several years of laparoscopic work.

\section{Acknowledgement}

The study is part of the "Industry on Campus" project IOC-103 (Ministerium fuer Wissenschaft, Forschung und Kunst Baden Wuerttemberg, initiated by Interuniversitaeres Zentrum fuer medizinische Technologien StuttgartTuebingen) "Interaktionsbasierte manipulatorgestützte Assistenz" in cooperation with TRUMPF Medizin Systeme and FESTO.

\section{Bibliography}

[1] Radermacher, K., Pichler, C. V., Rau, G.: Aspects of ergonomics in Minimal-Invasive Surgery - Analysis and Approaches, Engineering in Medicine and Biology Society, 1992 14th Annual International Conference of the IEEE, vol.4, pp. 1564-1565, Oct. 29 - Nov. 1, 1992

[2] Patkin, M., Isabel, L.: Ergonomics and Laparoscopic general surgery, in Laparoscopic abdominal surgery, (Graber, J. N., ed.), pp. 83-86, Kingsport: Arcata Graphics, 1993

[3] Miller, K. et al.: Ergonomics Principles Associated With Laparoscopic Surgeon Injury/Illness, Human Factors, vol.54, no.6, pp. 1087-1092, December 2012

[4] Pfeffer, S., Maier, T.: Analyse der System-Usability komplexer Mensch-Maschine-Systeme mit iFlow, 59. Kongress der Gesellschaft für Arbeitswissenschaft, pp. 681-684, Feb. 27- March 1, 2013

[5] Hart, S., Staveland, L.: Development of NASA-TLX (Task Load Index): Results of empirical and theoretical research, in Human mental workload, (Hancock, P., Meshkati, N., eds.), pp. 139-183, Amsterdam: North Holland, 1988

[6] Kuorinka, I. et al.: Standardised Nordic questionnaires for the analysis of musculoskeletal symptoms, Applied Ergonomics, vol.18, no.3, pp.233-237, Sept. 1987 